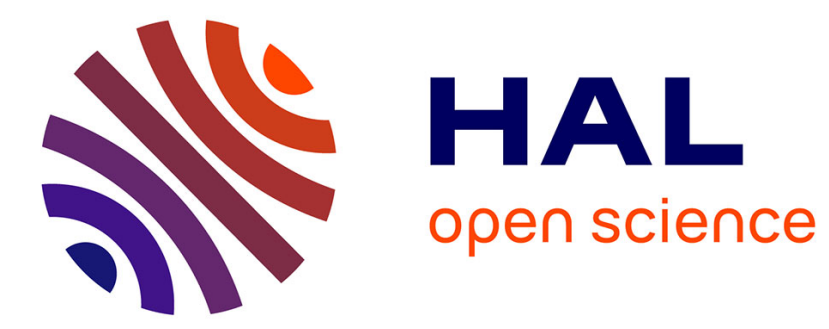

\title{
Colères et ordo amoris dans les styles rock
}

Jean-Marie Seca

\section{To cite this version:}

Jean-Marie Seca. Colères et ordo amoris dans les styles rock. Sociétés - Revue des sciences sociales et humaines, 2012, 117 (3), pp.15-33. 10.3917/soc.117.0015 . hal-03005627

\section{HAL Id: hal-03005627 \\ https://hal.univ-lorraine.fr/hal-03005627}

Submitted on 18 Nov 2020

HAL is a multi-disciplinary open access archive for the deposit and dissemination of scientific research documents, whether they are published or not. The documents may come from teaching and research institutions in France or abroad, or from public or private research centers.
L'archive ouverte pluridisciplinaire HAL, est destinée au dépôt et à la diffusion de documents scientifiques de niveau recherche, publiés ou non, émanant des établissements d'enseignement et de recherche français ou étrangers, des laboratoires publics ou privés.

\section{(이)(\$)}

Distributed under a Creative Commons Attribution - NonCommercial - NoDerivatives| 4.0 


\section{Colères et ordo amoris dans les styles rock}

Jean-Marie SECA ${ }^{1}$

Résumé : Le but de cet article est d'interpréter le sens socio-anthropologique de l'énergétique et de l'esthétisation de la colère dans le rock. On définira d'abord le rock, au sens large, de façon structurale et par son état d'esprit créatif, puis, la notion d'esthétique de la colère ; la pulsion colérique sera conçue comme un phénomène devant être nécessairement sublimée pour entrer sur la scène sociale. Puis, nous verrons dans quelle mesure cette sublimation de la colère s'articule à une philosophie de l'ordo amoris. Ce qui reconfigurera les fonctions et le sens de la rage dans l'expression et la création rock. Nous aborderons, dans une troisième partie, quelques exemples illustratifs.

Mots-clés : Rage, sublimation, musique rock, fétichisme, ordo amoris

Abstract: The purpose of this article is to interpret the socio-anthropological meaning of the energetic and of the aesthetic process of anger in the rock music. We shall define, at first, the rock music, in the broad sense, in a structural way and by its creative state of mind. Then, we shall speak about the notion of aesthetics of the anger; the choleric energy will be conceived as a phenomenon that must be inevitably sublimated to enter on the social scene. Secondly, we shall see that this sublimation of the rage is structured around a philosophy of the ordo amoris. This latter point will permit to re-configure the functions and the structure of the rage in the expression and the rock music's creation. We shall analyze, in the third part, some illustrative examples.

Keywords: anger, sublimation, rock music, fetishism, ordo amoris

La rage, au sens général de ce mot, serait l'un des deux constituants énergétiques du lien social, permettant l'émergence de l'envers, souvent symbolisé et scénarisé dans des rituels initiatiques ou des fantasmagories du merveilleux, du vivre-ensemble alternatif et d'un certain rapport à la transcendance et à l'étrange (Sloterdijk, 2007). Traquer ses formes cachées ou visibles et ses transmutations relève donc d'une nécessité pragmatique et sociologique, étant donné le contexte mondial d'atomisation croissante des revendications et des sentiments minoritaires. L'autre carburant de la viscosité des groupes et des relations interpersonnelles est bien entendu la libido qui est aussi définissable comme une quantité

\footnotetext{
1 Professeur de sociologie, Laboratoire Lorrain de Sciences Sociales (2L2S), Université de Lorraine; courriel: secajm@yahoo.fr.
} 
d'énergie sexuelle finalisée, circulante et/ou entravé/canalisée (Moscovici, 1981, pp. 322-334 ; Laplanche et Pontalis, 1967, pp. 224-228). Carl Gustav Jung y voit une énergie psychique, un appetitus (Jung, 1964, pp. 206-208). La « pulsion colérique », telle que nous la définissons ultérieurement dans ce texte, pourrait donc être incluse dans la métapsychologie jungienne de la libido.

La colère est notamment une manifestation de la tendance mimétique et ritualiste des masses (Girard, 1972, pp. 213-248). Comme la libido, en tant que forme passionnelle, elle ne peut s'exprimer sans être poétisée, parlée, sublimée et esthétisée parce qu'elle prend sa source dans une dynamique pulsionnelle innommable et dans la nécessité de sortie hors de soi ou du groupe dans lequel elle macère. Dans son acception esthétisée, voire parfois aseptisée, elle imprimerait une «marque de fabrique » à de nombreuses productions rock. Elle affleurerait aussi tendanciellement dans la configuration de certains styles et durant la réception des contenus-objets musicaux et leur appropriation par des publics.

\section{Le statut de la colère dans le rock}

Tout d'abord, quelle signification attribuera-t-on au terme « rock » dans cet article ? Nous le définirons intentionnellement dans une acception structurale et, en tant qu'état d'esprit pouvant comprendre, même si cela peut surprendre les puristes, le rap, le reggae et les formes postpunks. Seront donc incluses, sous ce terme générique, les tendances dites « industrielles », « ambient » (Toop, 2008, pp. 167-204) ou metal, aux ramifications hybrides (Mombelet, 2009). De plus, les musiques électroniques de Détroit, de Chicago, de Berlin, de Londres et de diverses régions d'Europe, inspirées ou non par des tempos dance ou rythm and blues prolongent et transforment des expérimentations sonores, aux origines plurielles, souvent rock ou soul, des années 1970 et 1980. Le rock lui-même, durant les années 1950, était une mixture de rythm and blues électrifié, associée à des stylistiques bluegrass ou country, ensuite rediffusée, repérée publicitairement au moyen d'une structure rythmique et mélodique conventionnelle mais susceptible de variations par rapport à la « norme », qualifiée durant un temps mythifié et court, de 1951 à 1957-1958 (Daufouy et Sarton, pp. 13-48), aux États-Unis, de « rock'n'roll ».

Tous ces mouvements globalement «rock», mais très variés dans leurs stylisations et leurs inspirations, ont été profondément marqués par une manière de produire, de créer et par des processus de fusion entre musiques dites «noires » et influences dites «blanches » ou « européennes ». La clé d'appréhension de ces styles est donc dans leur «textures » d'effets croisés, d'appropriations, de conversions et d'évitements interculturels, par contre-influences, imitations, transformations, rejets, critiques, mélanges (Hebdige, 2008, pp. 49-74). L'un des processus moteurs de ces interférences et de 
Seca Jean-Marie, 2012b, « Colères et ordo amoris dans les styles rock », Sociétés, n 117/3, (septembre-décembre), pp. 15-33.

ces « intermusicalités ${ }^{2} »$, souvent rugueuses, ayant donné lieu aux formes rock contemporaines, divisées en autant de branches et de styles qu'il y a de groupes ou de sous-groupes pour les créer, est le processus de catégorisation intergroupale 3 (Doise, 1979). La catégorisation intergroupale et sa stylisation sont des processus essentiels de construction de ces espaces culturels et sociaux. Si on se base sur cette spécificité psychosociale et culturelle des styles rock, il semble alors pertinent de lire, dans la matérialité et l'arrangement de leurs contenus musicaux, la cristallisation de la course à la différenciation sociale par la musique.

Nous abordons donc le rock, dans cette contribution, comme une structure sociologique et productive efficace, en tant que musique de masse (Denis-Constant, 2006, pp. 132-134) et comme label stylistique approximatif, évoquant un «état d'esprit». D'un point de vue structural et dans le faire, «rock» et «techno » ou «rap » procèdent d'une même mécanique créative, de confrontation intergroupale, émergente et comparative : traductions d'une rage de créer, visée de bricolage et utopie DIY (Do It Yourself), transformations des entraves ou contraintes en chants colériques ou très doux ou encore en musiques hallucinées sans texte, autoproduction, mode de vie alternatif, recherche nécessaire d'innovation pour être présents sur les marchés, stylisation et stratégie de visibilité. Les autres grands genres (musiques classiques ou contemporaines, jazz, du monde, formes folkloriques, variétés, revival

\footnotetext{
${ }^{2}$ Le terme « intermusicalité » est introduit pour résumer un mouvement complexe d'inspiration-expiration-reformulation du contexte socio-historique d'un musicien, de ses œuvres antécédentes, de modèles valorisés et de leur encastrement subtil dans ses productions actuelles. II présuppose aussi une interinfluence entre groupes et artistes. Laure Ferrand décrit très bien ce processus en évoquant une interaction allant de la passion initiale, son insertion sociale, à l'émergence d'une scène musicale ou d'expertises chez les fans, d'engouements autour d'artistes éponymes (Ferrand, 2010, pp. 203-271). Cette notion d'intermusicalité concerne cependant l'acte de composition et création. Elle est analogue à celle d'intertextualité, décrite notamment par Julia Kristeva et d'autres spécialistes de la littérature, au sein de la revue Tel Quel et dans diverses contributions qu'ils seraient trop long et malaisé de citer dans cet article. L'intertextualité fait référence à la mise en contexte historique, sociale, politique, stylistique (époque, esprit du temps) et textuelle (autres écrits, influences, inspirations) d'une création littéraire ou d'une fiction. Par extension, on peut considérer que l'intermusicalité définit la musique d'un groupe ou d'un créateur en tant que structure réticulaire interconnectée à d'autres œuvres et aux contextes de sa production. Sophie Stévance utilise la notion d'intertextualité musicale pour analyser les reprises, réactivant le sens de chansons d'une figure féminine de la chanson des années 1930, Mary Travers, au Québec. Ainsi, des artistes québécoises et canadiennes contemporaines, y compris des musicienne anglophones, expriment une esthétique commune en se référant à cette source prisée de la musique populaire (unité), tout en déclinant leur propre vision de l'engagement artistique à travers leurs actualisations, citations et hommages (éclatement) de cette même œuvre (celle de Mary Travers dit « La Bolduc » : Stévance, 2010, p.71).

${ }^{3}$ Ce processus intergroupes de définition de soi, impliquant une survalorisation de la production propre et se faisant en opposition à un autre ensemble groupal en comparaison avec soi et son propre groupe, a été (et est) extrêmement étudié en psychologie sociale, principalement par des auteurs comme Willem Doise dont on cite la référence (ouvrage collectif portant sur tous les aspects de ce même phénomène) en bibliographie. Mais on peut se référer à une liste de nombreux auteurs dont essentiellement Henri Tajelel et John Turner, représentants de l'école dite « de Bristol », qui ont longuement et pertinemment théorisé et expérimenté sur cette mécanique de construction des identités, inhérente à tout groupe social.
} 
populaires) sont aussi partiellement marqués par ce mode de production. Mais aucune autre tendance que le rock et les styles connexes contemporains n'a mieux exprimé les turbulences et tumultes des foules et des fractions ou « cristaux de masse », depuis environ soixante-dix ans. On fera remonter l'état d'esprit (et le «moulage ») du rock à un contexte historique marqué par l'émergence des premières formes de minimalisme technologique, productif, allié à un modèle commercial et culturel spécifique et notamment à la grande grève des musiciens d'orchestre, aux États-Unis, durant les années 1940 (Buxton, 1985, pp. 36-38). À ce moment-là, l'industrie du disque et un simple raisonnement financier influencèrent les intentions créatives et l'impact des musiques électrifiés et des petites formations qui pulluleront, tant dans le be-bop que dans le futur rock'n'roll des années 1950. Pour caricaturer, on peut presque dire que les rocks vont résulter d'une réponse des minorités artistiques et des majors du disque à un problème d'employabilité et de recherche de profit, avec le minimum de musiciens, plus ou moins doués pour leur art. Plus subtilement, on pourrait dire que les majors du disque et les minorités rock ont exploité intelligemment les trésors de colère des masses, aux États-Unis, puis en Europe. C'est pourquoi, malgré l'ancienneté des phénomènes de catégorisation intergroupes et de guerre symbolique par l'esthétique, le rock, au sens extensif du mot ${ }^{4}$, contient et récapitule, en dépit de ses effets pacificateurs et cathartiques sur les jeunes particulièrement, l'essence de la colère dans sa dimension de canalisation du mimétisme et de besoins de comparaison sociale.

L'obsession mimétique et comparative n'a certes pas attendu la diffusion de ces tendances musicales pour exister. Cependant, l'histoire même des tensions intergroupes aux États-Unis (luttes raciales notamment), terre d'élection du blues et du rock'n'roll, ainsi que de nombreux autres courants, le démontre avec force. L'impact des appropriations identificatoires dans les relations musiciens / publics (Chastagner, 1998, pp.117-143) le confirme. Il y a enfin d'autres éléments de mimétisme et de violence latente qui expriment cette rage latente. Ils sont associés à la fonction politique et d'expression de lutte des classes de ces musiques. Toutes ces caractéristiques indiquent que l'approche des formes et styles rock par leur granulation colérique est heuristique (Seca, 1988, 2001, 2007).

\footnotetext{
${ }^{4}$ Nous avons conscience de la provocation que recèle notre position structurale, faisant fi des nuances stylistiques propres à chaque courant, comme le rap et le reggae ou d'autres formes musicales majeures. Mais nous visons un raisonnement sociologique général et une approche structurale du phénomène d'émergence rock et non un épluchage « style par style » des traductions des rages en esthétiques (ce qui dépasserait l'objectif de cet article).
} 


\section{Les routes entravées du sublime}

Cela dit, s'il y a plusieurs chemins pour parvenir au sublime, il y a aussi diverses routes pour exprimer l'enragement. La séparation du psychisme en deux instances (conscient/inconscient; rationalité / mysticisme, répondant à des disjonctions comme «ordre / désordre » ou « réalité / merveilleux »), qu'on la considère comme une métapsychologie inacceptable ou qu'on la prenne comme une référence incontournable des sciences humaines (Anzieu et al., 1974 ; Freud, 1982), concernerait probablement aussi l'énergétique de la colère. Pourquoi mettre en avant cette séparation ? Tout simplement parce que le sublime résulte de l'envie de recoller ces deux espaces dans l'esprit des artistes.

Beaucoup de spécialistes plaident pour une approche historique de l'inconscient (notamment Dufour, 2007, pp. 298-326). Et l'on peut retracer aussi une histoire sociale des modes d'expression des colères qu'on peut intégrer dans ce continent-là, souvent décrié dans l'approche en sciences humaines. Approchons, pour l'heure, la question sous un angle de vue structuraliste. Pour que les conduites concrètes des artistes trouvent leur expansion créative efficiente, leur espace privé entravé (névrotique et familial, contraint socialement et culturellement, interconnecté avec des réseaux, etc.) doit être articulé à l'imaginaire et aux matrices culturelles de leur espace sociétal et urbain d'existence. Cet imaginaire et sa cohorte d'images et de représentation, associées à des pratiques et rituels groupaux et de masse, forment l'essentiel de l'inconscient au sens large de ce terme. Tout en étant relié à un contexte et associé à une socialisation primaire spécifique, ce domaine privé entravé demeure la source principale de la fantaisie créative. L'espace privé entravé permet d'intérioriser l'extériorité sociale et d'extérioriser l'intériorité et l'intimité des fabricants d'esthétiques. En ce sens, il est le lieu d'émergence d'un imaginaire personnel et donc de l'originalité d'un créateur et aussi des passions d'amateurs d'un genre qui se greffent sur ces formes semi-intimes, semi-publiques. II s'agit donc d'un dispositif artificiel, voire virtuel (« forme sociale 2 » dans le schéma ci-dessous). II correspond à une « bulle » (pour une introduction à la théorie des sphères et bulles voir : Sloterdijk, 2010, pp. 33-91). Du fait de sa virtualité active pour le psychisme, on peut le qualifier aussi de «forme commune possible » ou de « fétiche » (Assoun, 2006 ; Rosolato, 1967, p. 20). II cohabite efficacement avec les pulsions, le besoin d'autoprotection et les méandres des biographies. On doit le voir, par conséquent, comme une forme dérivée, consécutive, un déroutement. On pourrait dire qu'il s'agit d'un « accident de la satisfaction libidinale », d'une dérivation acceptable et donc d'un malaise dans l'esthétisation du désir. Freud rappelait, il y a plus de quatre-vingts ans, qu'« un seul point semble certain, c'est que l'émotion esthétique dérive de la sphère des sensations sexuelles ; elle serait un exemple typique de tendance inhibée quant au but » (Freud, 1970, p. 26.) Ce dispositif ou cette forme commune possible condense et articule donc, via une déviation névrotique, des inspirations 
artistiques et les émotions les plus aberrantes et personnelles. Issue du domaine privé des créateurs, ce dispositif esthétique germinatif est, par conséquent, le point de concentration d'une dérivation partielle de l'énergie psychique (libidinale et passionnelle / rageuse) et, parallèlement, d'activation et d'utilisation de structures signifiantes archaïques et collectives (« formes sociales 1 », dans le schéma ci-dessous).

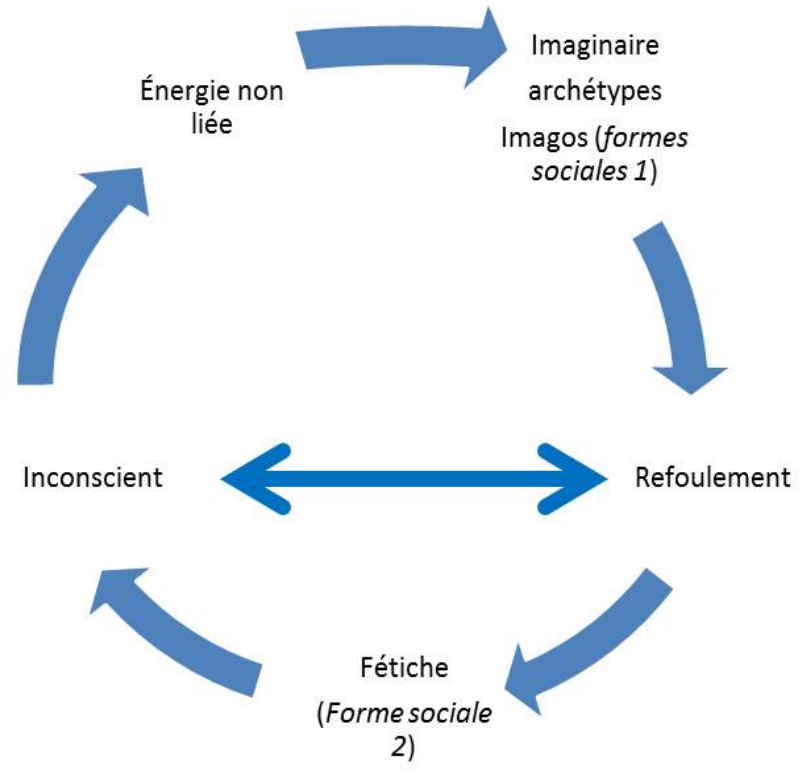

Schéma 1 : Économie libidinale d'un fétiche ou du dispositif esthétique

On arguera, dans cet article, que la sublimation ne consiste pas uniquement à dévier de leur objet (primaire ou imaginaire) de l'énergie libidinale, mais aussi de la violence et des pulsions destructrices ou haineuses. La sublimation colérique conduit à une sorte de décharge instable d'une énergie par ailleurs esthétisée, dérivée, ritualisée, se traduisant en effets de transes. Dans ce cas-là aussi, ce processus cathartique ne peut avoir lieu qu'à la suite de la confection d'un dispositif spécifique, d'un fétiche (ou «forme sociale 2 », dans le schéma 1 précédent), favorisant la « séance de décharge ». Par le passé, l'expression « refoulement de la révolte » a déjà été suggérée pour décrire ce mouvement de sublimation de la rage et des frustrations chez les musiciens underground (Seca, 2001, pp. 204-211). La sublimation présuppose la prégnance d'une instance sociale contraignante et donc du mécanisme de refoulement qui « se produit dans le cas où la satisfaction d'une pulsion - susceptible de procurer par elle-même du plaisir - risquerait de provoquer du déplaisir à l'égard d'autres exigences [...]. II peut être considéré comme un processus psychique universel en tant qu'il serait à l'origine de la constitution de l'inconscient comme domaine séparé du reste du psychisme » (Laplanche et Pontalis, 1967, p. 392). 
Le refoulement, issu des effets de contrainte sociales, économiques, historiques spécifiques, et donc d'entraves plus ou moins différentes, selon les individus empêchés, permettrait donc de reproduire de l'inconscient contextualisé, historicisé et individualisé du fait de son émergence esthétisée et de son arrimage aux imaginaires sociétaux. C'est un espace topologique, symbolique et expressif, rendant possible, accessible et diffusable socialement une sorte de «langage autre » sur le monde et sa foisonnante réalité. L'inconscient n'est pas si mystérieux à saisir et à détecter qu'on le croit. Il est devant notre nez. II faut juste savoir l'observer comme un condensé d'imaginaires et de représentations. C'est pourquoi il s'agit d'une structure assez modulable en fonction des contextes qui renvoient à autant de groupes ou d'individus producteurs d'esthétiques.

Mieux vaut le redire : la sublimation, en tant que telle, serait une sorte de mécanisme aberrant, l'équivalent d'une tumeur maligne, permettant de faire ressortir, métamorphosées, ces énergies non liées et ces ruminations, provenant de tensions au sein de l'espace privé entravé. Elle permettrait de canaliser et d'organiser ces émotions autour d'un fétiche en puisant des contenus dans des ressources imaginaires déjà existantes comme des imagos ${ }^{5}$, des archétypes et des représentations socialement diffusables et inscrites sociotemporellement. Ainsi, par l'activité de sublimer, on sacrifie une partie de soi (mécontent) au profit d'un soi socialisé et socialisable. De façon pragmatique, l'engagement dans une activité artistique conduit à différer la «sortie » de la violence et de la morosité au profit d'activités plus socialisables. La révolte, objet d'un refoulement, lorsqu'elle est transmuée en musique, serait alors convertie en structures signifiantes favorisant, par exemple, l'expression et la gestion d'états de transes et des « séances de décharges » (états de transe). Ces formes esthétiques cristalliseraient aussi les méditations envieuses de l'admirateur ambivalent qu'est tout membre d'une masse. En un mot comme en cent, les musiques forment des structures de retenue, de transformation et de cristallisation des colères.

\footnotetext{
${ }^{5}$ L'imago, notion issue de la psychologie jungienne, est une survivance imagée de situations, figures (parentales) et conflits psychiques vécus durant la phase enfantine du complexe d'Erdipe (Laplanche et Pontalis, op. cit., pp. 196-199). II s'agit donc d'une sorte de «représentation inconsciente » ou de « schème imaginaire », liés au conflit œdipien, par lequel un individu tente d'appréhender autrui.
} 
Qu'est ce qu'une esthétique de la colère?

Postulat: la rage (comme la libido) ne peut s'exprimer sans être esthétisée et traduite
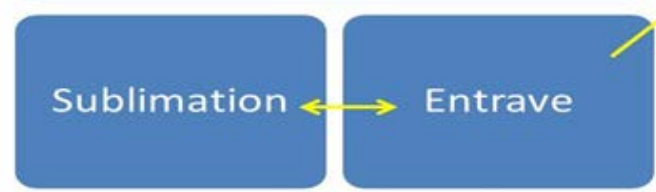

Stylisation

(exhibition,

fétiche)

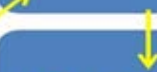

Rêve éveillé,

transes

Schéma 2 : Entrave et circuits esthétisés de la colère

Theodor Adorno ou Frantz Fanon avaient déjà perçu un lien critique entre l'expérience de la domination ou de l'aliénation et la recherche de la transe (Fanon, 1961, pp. 87-88 ; Adorno, 1950, 1971, 2001). Comme l'aliénation, la colère pourrait donc aussi être sublimée, exhibée et procurer un apaisement à son créateur et ses spectateurs. Elle serait accolée à un « rêve de remplacement » et à une forme subtile, socialement diffusable et acceptable de névrose esthétisée. Cette dernière correspondrait à un état de prépossession. La forme esthétique condense alors les émotions et les rages. Elle contient des parcelles de l'énergie négative ou positive antécédente tout en exprimant une idéalisation de soi, en réaction à une révolte non aboutie et à une domination frustrante. Le sublime console donc l'individu créateur ou spectateur en l'arrimant fermement à une forme identitaire (commune) possible, et donc à un fétiche idéalisé.

«L'artiste est, en même temps, un introverti qui frise la névrose. Animé d'impulsions et de tendances extrêmement fortes, il voudrait conquérir honneurs, puissance, richesses, gloire et amour des femmes. Mais les moyens lui manquent de se procurer ces satisfactions. C'est pourquoi, comme tout homme insatisfait, il se détourne de la réalité et concentre tout son intérêt, et aussi sa libido, sur les désirs créés par sa vie imaginative, ce qui peut le conduire facilement à la névrose [...]. II sait donner à ses rêves éveillés une forme telle qu'ils perdent tout caractère personnel susceptible de rebuter les étrangers, et deviennent une source de jouissance pour les autres. II sait également les embellir de façon à dissimuler leur origine suspecte. II possède, en outre le pouvoir mystérieux de modeler des matériaux donnés jusqu'à en faire l'image fidèle de la représentation existant dans sa fantaisie et de rattacher à cette représentation, existant dans sa 
fantaisie inconsciente, une somme de plaisir suffisante pour masquer ou supprimer provisoirement du moins, les refoulements » (Freud, 1982, p. 354.)

Le processus de refoulement, celui de constitution d'espaces intimes entravés, potentiellement communs et l'accès aux états mentaux fournis par les formes esthétiques sont profondément intriqués. La transe, comme jubilation et comme sensation plus violente (possession, obsession, expériences corporelles extrêmes), est alors une réappropriation paradoxale d'un pouvoir sur soi (ses propres désirs et sentiments) par une sortie « hors de soi » et une identification à un « monde commun possible » qu'est toute œuvre d'art et à laquelle on finit par penser qu'on ressemble forcément.

«Lorsqu'il a réussi à réaliser tout cela, il procure à d'autres le moyen de puiser à nouveau soulagement et consolation dans les sources de jouissance devenues inaccessibles, de leur propre inconscient; il s'attire leur reconnaissance et leur admiration et a, finalement, conquis par sa fantaisie ce qui, auparavant, n'avait existé que dans sa fantaisie : honneurs, puissance et amour des femmes » (ibid., pp. 354-355.)

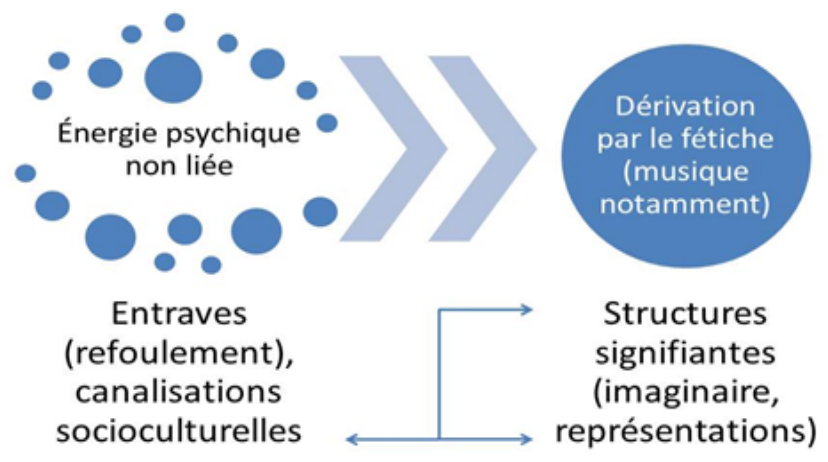

Schéma 3 : Énergie psychique non liée, fétiche et imaginaire

On l'a déjà écrit plus haut : d'un point de vue théorique, l'inconscient regroupe l'ensemble des pulsions, que celles-ci soient agressives ou amoureuses. L'énergie non liée qui y circule, passe ensuite par des entraves, des barrières qui augmentent parfois la pression énergétique pour que « ça sorte ». Ces entraves sont aussi parfois des dérivations, donc des canaux de sublimation impliquant différents types de symbolisation dont certaines sont organisées autour du ressentiment ou, pour le dire autrement, d'effets proprement et positivement pervers. 


\section{Ordo amoris et rage}

Les émotions colériques ne peuvent donc surgir publiquement, à l'état brut, sauf en prenant un masque qui les parodie et les camoufle. Ce thème du masque et de l'émergence publique de la persona est très heureusement traité par Michel Maffesoli dans son livre sur le temps des tribus (Maffesoli, 1988) et par des spécialistes des musiques metal où l'effet de scène et le transformisme, avec l'usage des corpses paints, dominent (Mombelet, 2009 ; Walzer, 2007). La violence directe n'a aucun intérêt en tant qu'objet d'étude esthétique. Les états colériques et le ressentiment doivent, pour trouver une destination, être transformés et exhibés à travers une codification, comme tout état pulsionnel, même si on perçoit une fine frontière entre violence pure et ritualisation esthétique dans certains styles ou certaines œuvres. Roland Barthes, dans son livre, Fragments d'un discours amoureux, avait bien montré comment la poésie et les lettres d'amour les plus exaltées naissent de dépits les plus solitaires (Barthes, 1977, p. 115). Les esthétiques de la colère ne sont pas de nature très différente. Robert Sternberg, a lui-même théorisé un modèle de la haine, homologue à son triangle de l'amour (Sternberg R., 2005 ; Sternberg R. et Sternberg K., 2008). Pascal Bruckner va même plus loin en supposant que les idéologies et les religions de l'amour pourraient bien être à l'origine des totalitarismes contemporains et des excès de haine les plus ravageurs (Bruckner, 2009, pp. 225-267). Sloterdijk abonde dans ce sens en désignant notamment Aurelius Augustinus comme le « père de la Terreur » (Sloterdijk, 2007, p. 135). Les doctrines de l'amour sont alors parmi les premières formes de justification des punitions infligées, au nom du bien pour tous et de sentiments élevés, aux hérétiques comme aux infidèles. Pour le philosophe allemand contemporain, on détecte des « archives de la colère » dans l'histoire millénaire des révoltes humaines et des textes de l'Ancien Testament (ibid., pp. 100-152).

Cependant et complémentairement, il y a un versant tonifiant et créatif de la colère et de l'expressivité enragée qui exprime une sorte de profonde intuition du temps et des influences sociales. C'est par cette tonicité anticipatrice et cette vitalité mobilisatrice que les styles rock sont aussi des esthétiques de la colère. L'enragement rock et même le punk quand il ne prévoyait aucun avenir, en 1976, en sont de beaux exemples. L'impact des rages sociales et biographiques affleurant dans ces styles soutient donc celui d'un ordo amoris (Scheler, 1996, pp. 54-88; Maffesoli, 2007). L'effet du fétiche musical, élaboré dans ces moments de gobage esthétique, est aussi en relation avec la fonction des devises chez les producteurs et créateurs (Seca, 2009, pp. 19-21). En effet, l'atteinte d'un ordo amoris est assez proche de ce que nous nommons l'assomption d'une devise ou d'un PVI (projet viable d'identification) dont les caractéristiques sont la plasticité, l'évolutivité, la progressivité, et l'accomplissement de soi (Seca, 2010, p. 73). Une illustration philosophique de ces phénomènes est la notion de Vorbildmodelle ou modèle-type (Max Scheler, 1958, p. 17). II s'agirait d'un état mental, culturel et d'un processus (presque ascétique) 
permettant une « vertébration » de la personne face au désordre initial de la psyché et du cœur. La notion de devise recouvre le même champ de conduites d'autoreconnaissance du créateur dans le miroir (modèle plus ou moins inspiré) de son œuvre. Elle favorise sa « navigation philosophique et existentielle » dans la création et l'avènement progressif d'une forme. Elle autorise surtout une compréhension du politique soit en tant qu'émergence autonome d'un répertoire propre d'un artiste, soit comme contreréaction à un modèle " plaqué ", imposé par des partis ou des ordonnateurs d'idéologies. Paradoxalement, la devise ne peut être issue que de cultures et conduites anomiques, hors convention et sortant du lot du convenu et du convenable. C'est ce que nous avons toujours défendu, depuis le début de nos recherches sur les musiques populaires, en 1982-1987.

C'est aussi pour ces raisons-là que les styles rock offrent une matrice adaptée, large, ambiguë de codification des états colériques et du mécontentement. Ils favorisent, mieux que d'autres formes musicales, les investissements déceptifs ou les contre-dépendances face aux frustrations et aux dominations sociétales. Peut-on y observer des «cultures du ressentiment organisationnel et postmoderne » (Seca, 2010, 203-254) ? La forme rageuse des styles rock doit notamment être décrite dans ses imaginaires soniques et dans ses effets psychosociaux et représentationnels sur l'auditoire, voire dans la fascination pour certaines idéologies prônant des modes de vie utopiques ou réactionnaires, voire régressifs. Le contenu stylistique (rythme, types de messages, mythes, représentations, présentation sur scène) a certainement aussi une grande importance. L'imaginaire de nombreux styles musicaux électro-amplifiés fournit divers exemples du ressourcement de leurs publics et producteurs successifs aux « archives de la colère » (Sloterdijk, op. cit., pp. 111-112 et 194-196). II est d'ailleurs intéressant de savoir que c'est le modèle d'archivage des arts (muséologie notamment) qui est appliqué aux formes colériques par ce même auteur (ibid.). Avec l'industrie du disque et du son, l'archivage des colères esthétisées rock est devenu une quasi-science à tel point que certains jeunes sociologues des musiques populaires confondent « connaissance des styles musicaux » et « sociologie ». Le registre dit de l'« amour » ou de la sympathie n'est donc probablement pas la seule grille interprétative de ces cultures. L'étude des relations intergroupes indique que l'on n'est intégré dans un groupe tribal, fusionnel, bienfaisant, en sympathie réciproque que par contre-effet sociétal et par opposition à d'autres tribus, ensembles, structures qui représentent l'ennemi, l'antithèse, l'antimodèle. L'amour et le registre de l'amour se vivent comme des «drôles de bulles » et ils sont contrebalancés, depuis des millénaires, par des violences avérées ou symboliques contre les barbares ou divers boucs émissaires menaçant, réellement ou imaginairement, de les faire exploser. La conception interculturaliste et rugueuse de l'émergence stylistique rock est donc inextricablement en lien avec une conception sociopolitique et artistique de l'authenticité. L'authenticité expressive (le «vrai » soi) est alors devenue une norme 
culturelle de l'expressivité et de la créativité très sensualiste dans ces musiques et de chaque groupe ou producteur en compétition ou en situation d'inter-influence agonistique. Elle se traduit aussi par une nécessité d'imposition d'un soi plus normativement rock que rock. Et elle implique donc de voir dans la devise une sorte de propagande allant du soi authentique à autrui.

Dick Hebdige a insisté sur le caractère dialogique, conflictuel et intercommunautaire de la genèse des divers styles musicaux, en Angleterre, durant les années 1970. Nos enquêtes empiriques l'indiquaient aussi pour les groupes amateurs parisiens alternatifs (Seca, 2001, 2007). Le punk anglais en fut un exemple clair (Hebdige, 2008). Les styles jamaïcains et antillais (rocksteady, ska, reggae) constituaient des modèles comparatifs poussant notamment les punks au métissage musical et à un universalisme culturel radical, teinté de cynisme rageur. Ce rapport dialogique aux autres groupes sociaux a cependant conduit une partie de la jeunesse blanche anglaise à l'isolement populiste, par exemple, dans certaines ramifications de skinrock aux valences politiques variables et extrémistes (voir les travaux de Gildas Lescop à l'université de Nantes). Cette conception interculturaliste ne mène pas systématiquement au dialogue structuré et au métissage harmonieux des cultures. En fait, elle produit des routes divergentes vers le sublime et donc aussi des stylisations enragées ou colériques, parfois haineuses. La machinerie de création interculturelle et intergroupe serait plutôt une sorte de « marque de fabrique » du rock. Même les musiques les plus planantes et apaisantes n'existent que par rapport à un environnement stressant ou violent. Le plein exprime le creux. Le relaxé dévoile l'énervement qui le précède ou lui succède. L'intensité d'un rythme compense la sérénité, trop fortement exhibée, du quotidien et de son confort. Retracer et pister les traductions colériques signifient donc, tout autant, appréhender les formes frustrées, ennuyées et révoltées dans les musiques dites « politisées » ou extrêmes, que de jauger la nature et le sens des distractions dans ce qu'elles ont de plus détaché par rapport aux ambiances sociopolitiques environnantes.

\section{Quelques exemples illustrant notre argumentaire}

Une très belle analyse de Jeremy Price montre la permanence de l'impact enragé d'un morceau, initialement composé dans une prison du Sud des États-Unis. En retraçant l'histoire d'un standard, « Rock Island Line », dont l'un des premiers rockers anglais, Lonnie Donegan, a été le porte-son et le porte-voix, on peut suivre une ligne rebelle transculturelle qui prolonge la voie de chemin de fer (Rock Island Lines), empruntée par des jeunes Noirs américains décidés à en finir avec la galère, voulant s'échapper des prisons, des lourds travaux de bucheronnage et des champs de coton du Sud des États-Unis (Price, 2010). Ce tube du rock britannique, sorti quelques mois après « Rock around the clock», se vend, en 1955, à plus de trois millions d'exemplaires en six mois. Cette chanson, provenant du pénitencier 
d'Angola, en Louisiane, a été écrite par un ex-détenu, Lead Belly, et enregistrée, en 1934, puis diverses fois jusqu'en 1946. II s'agirait originellement d'un chant de travail afro-américain (bûcheronnage, ramassage de coton) et / ou d'une chanson de chemin de fer, donc d'ivresse du voyage. II va sans dire que cet exemple illustre, à lui seul, la notion d'intermusicalité et celle de rage transmissible par l'esthétique, telles que nous les avons décrites théoriquement au début de cet article. Tout un bouillonnement culturel dans l'Angleterre des années 1950 explique l'accueil très favorable de cette chanson de chemin de fer, venue d'outre-Atlantique et le succès de Donegan aux États-Unis avec sa reprise. L'évolution des modes de vies et de consommation britanniques avait engendré une diffusion massive de ces styles. L'émergence des teenagers, durant la même période historique qu'aux États-Unis, faisait de la Grande Bretagne un terreau propice aux germinations rock. Price explore une métaphore (le symbole du train et de sa vitesse), très bien musiquée dans « Rock Island Line », qui fut aussi la première ligne de chemin de fer traversant le Mississipi au XIXe siècle, figurant l'émancipation, initialement des africains américains ou de prisonniers. Étrangement, ce morceau, issu de prisons et de la protestation blues, fera écho, vingt ans plus tard, aux révoltes consommatoires et aux désirs d'émancipation des jeunes Britanniques face à l'emprise des privations d'après-guerre et aux traditions, par l'adoption musiquée de la symbolique moderniste du train. L'écoute de ce morceau et de ses différentes versions, sa granulation émotionnelle, son énergie entrainante, permettront au lecteur de comprendre la signification de l'expression « archive transmissible de la rage ».

Contrairement aux prêt-à-penser sur leur cohérence et leur formalisation rituelle, il y a un vrai et houleux dialogue entre styles et donc entre ces contre- ou -sous-cultures. II serait faux de cantonner notre approche sur la colère au seul rock des années 1950. Les styles, si saillants et attrayants pour le regard sémiologique et dont on se repait pour établir un travail de terrain, sont issus de contre-stylisations et de reformulations. On peut prendre l'exemple du gabber belgo-hollandais et du nord de la France. Ce style électronique s'édifie sociopolitiquement et notamment contre le rap et certains textes provocateurs et enragés de ces derniers. L'importance de l'imaginaire colérique dans le hip-hop participe de cette contreinfluence interculturelle et catégorisante. On sait que l'expression fâchée est un des fondements stylistiques du rap, que ce soit dans le ton de la voix, dans les lyrics ou dans le style musical très haché et rapide. Le dénigrement de ces rappeurs fâchés par des gabberskins peut être observé sur divers sites web. Des travaux d'enquête exploratoires dans le nord de la France, sont édifiants (François, 2011). L'approche intertextuelle et interculturelle du rock, conseillée par Hebdige, fonctionne alors à plein régime. Le gabberskin et le rap ne sont pas directement et musicologiquement parlant du pur rock. Cependant, ils sont illustratifs d'une structure intergroupe des choix stylistiques et de l'affichage d'un hyper-formalisme 
fermé, impliqué et exprimé par la stylisation du rap comme du gabber. On pourrait faire le même type de remarque pour d'autres styles pop. Comme l'évoque très bien Hebdige dans son livre :

"Pour utiliser le lexique de la sémiotique, on pourrait dire que le punk "inclut" le reggae sous la forme d'une "absence présente", un trou noir autour duquel il articule son discours. Et d'un point de vue métaphorique, on pourrait étendre ce rapport à l'ensemble des questions de race et relations interraciales. C'est ainsi que nous pourrions faire l'hypothèse que la démarcation rigide de la frontière entre le punk rock et le reggae exprime non seulement une "crise d'identité" spécifique à la sous-culture punk, mais aussi les contradictions et les tensions plus générales qui inhibent le développement d'un dialogue ouvert entre une culture immigrante ayant un caractère fortement "ethnique" et la culture du prolétariat local pourtant censée "inclure"la précédente [...] » (op. cit., p. 72.) Un peu plus loin, le même auteur indique: "Peut-être que les différences excessives entre le langage rock et celui du reggae ne permettaient pas de créer une véritable synthèse. Le déphasage stylistique fondamental qui les caractérise - que ce soit au niveau du répertoire vestimentaire, de la danse, de l'expression verbale, de la musique, du type de drogue consommée ou de la trajectoire historique-, marqué par l'émergence de l'ethnicité noire dans le reggae, engendrait une dynamique particulièrement instable au sein de la sous-culture punk. C'est cette tension qui donnait au punk son look un peu rigide, son caractère bizarrement ankylosé, son "idiotie" spécifique, laquelle trouvait à s'exprimer silencieusement à travers les surfaces lisses de plastique et de caoutchouc et l'espèce de robotisme sado-maso qui incarne le signifiant "punk" aux yeux du monde. Figée dans une éternelle et glaciale paralysie, au cœur même de la culture punk, se dissimule la dialectique entre la culture blanche et la culture noire - une dialectique qui, au-delà d'une certaine limite (celle de l'ethnicité), s'avère incapable de se renouveler, prisonnière de sa propre histoire et de ses irréductibles contradictions (op. cit., p. 74.)..

Autre exemple: les symboles guerriers, mêlés aux imaginaires sataniques, païens, nazis ou nordiques, dans le metal européen, distillent une panoplie d'icônes colériques puissants et illustratifs. Les travaux de divers sociologues du metal, en France (op. cit.), ont pu permettre de détecter, dans certains « sous-styles » de ce genre, une adhérence aux idéologies et aux imageries flirtant avec le révisionnisme ou ce qu'on appelle trop pudiquement « une fascination pour le nazisme » ou des idéologies régressives. Mais il n'y pas que ces éléments. Toute la mise en scène metalleuse est organisée autour d'une figuration colérique, qui, il faut s'en souvenir, est assez fortement présente dans le panthéon des dieux germaniques, scandinaves et indo-européens, ainsi que dans de nombreux dossiers thymotiques ${ }^{6}$

\footnotetext{
${ }^{6}$ Le thymos renvoie à une étymologie grecque du terme « colère ». On doit y voir le sens de « fierté », de " dépassement de soi », d'« énergie vitale tendu vers l'accomplissement de soi » (voir Sloterdijk, op. cit.).
} 
répertoriant les remontrances du Dieu de l'Ancien Testament envers son Peuple. Avec quelques anges déchus, Satan, le sémillant « chargé de mission colérique », assumera seul, la gestion des vengeances et de l'enfer, à partir de la période des écrits des Apocalyptiques préchrétiens (Ile siècle avant J.- C.). On ne peut s'empêcher de faire le lien entre ces anciens « dossiers de la colère », les obsessions religieuses satanistes et la scénographie de l'enragement des producteurs de metal.

L'extrémisme sonique (vitesse, intensité, prise de drogue) et politique (résistance, zones autonomes temporaires, autogestion des transes tout autant que des sandwiches de l'after car l'accès aux transes fatigue et creuse les estomacs) des « technoïstes » des free-parties traduit une autre version de la rage, incarnant la libido dominandi, évoquée par Dany-Robert Dufour, dans un écrit discutant la position perverse dans la postmodernité (Dufour, 2009, pp. 15-17). Les musiques industrielles ont construit une série de productions radicales, en se centrant essentiellement sur un rapport exclusif et provocateur, voire mortifère, pour certains groupes, face à leurs publics. Pour illustrer cette schématisation, on peut citer le groupe de musique électronique extrême Whitehouse dont l'un des objectifs est explicitement de plonger le public dans un état avancé de soumission. II joue intentionnellement au-dessus de cent-vingt décibels (pour détruire les tympans des auditeurs ?). Les références philosophiques et artistiques des thèmes traités par ce groupe sont celles de la perversion (sadisme, crime, pédophilie) et de formes dites « sadiennes et raffinées de la violence "7. L'idéologie sadomasochiste dans les styles électroniques et les constituants imaginaires analogues dans les musiques gothic exemplifient les plis d'ambiguïté concentrés dans les formes rock (Rigaut, 2009, pp. 219-221). Ces sous-cultures voisinent avec des conceptions spécifiques du merveilleux et mettent en exergue un fétichisme explicite, exprimant d'ailleurs avec exactitude notre idée d'expressivité entravée.

Enfin, les musiques rebelles et politiques, comme l'Internationale dont la musique et les paroles ont été composées après la révolte de la Commune de 1870, la grille de lecture nietzschéenne de la tragédie grecque et l'histoire même des musiques protestataires ne sont qu'à peine effleurées dans ce texte. On sait que le travail esthétique sur le ressentiment fait partie du rock, dès le début de ce genre (voir Daufouy et Sarton, 1972 ; Delmas et Gancel, 2005, notamment).

\footnotetext{
${ }^{7}$ http://www.susanlawly.freeuk.com/textfiles/acephale.html: voici un extrait final d'interview sur ce site d'un musicien, Peter Sotos, ancien membre de Whitehouse : «-A (question à Peter Sotos): Tu as joué- tu joues avec Whitehouse. Rencontre \& intérêt pour Whitehouse?- Réponse de Peter Sotos: J'ai été en relation avec la Come Organisation [...] depuis pas mal du temps, et j'ai un immense respect pour William Bennett. Souvent, lorsque j'écris, j'utilise soit un titre, soit des lyriques de Whitehouse - nous avons beaucoup de goûts en commun. Whitehouse réussi à saisir la force, l'énergie et le désir que je trouve inhérent à la violence sexuelle et au sadisme extrême. Whitehouse est grandiose. De nos jours, le LP Great White Death est un grand classique ».
} 


\section{Conclusion}

Tous ces éléments concourent à offrir de l'émergence stylistique rock et de ses dérivés, l'image d'un témoignage mystique commercialisé sur le ressentiment et sur un mécontentement quasi métaphysique. II ne faut pas éluder l'importance de l'inscription de ces styles dans une structure culturelle plus large et ancienne, associée à une mémoire sociétale et historique intériorisée des frustrations et des violences passées. On peut même aller encore plus loin et mettre en connexion la matrice politique de l'Europe (voire même la structure culturelle de sa décomposition possible durant la crise de la dette souveraine et de l'euro de 2011-2012) et l'émergence des formes rock. On pourrait faire le même type d'hypothèse homologique entre les musiques rock ou industrielles, au Japon, et une mémoire diffuse des explosions nucléaires ou l'absurdité des concentrations urbaines hyperdenses. On dit souvent, par anti-européisme, qu'il n'y a aucun texte fondateur de l'Europe et de sa « nation multi-ethnique ». Ce texte existe cependant. II ne prend pas la forme d'un manifeste de la République fédérale d'Europe. II trouve ses sources souffrantes et amnésiques dans les archives du procès de Nuremberg, des camps de la mort, des massacres, des deux guerres mondiales et de l'angoisse retenue du long conflit entre les blocs communistes et libéraux. L'identité fondatrice d'une nation reposerait, selon Anne-Marie Thiesse, sur l'identification aux ancêtres, à un folklore et sur l'association de la culture nationale à une culture de masse (Thiesse, 1999, pp. 230-240). Or, l'imaginaire politique de l'Europe s'est construit à partir des trous béants des guerres et de la terreur glacée de l'équilibre entre puissances, menaçant de se détruire totalement. Ses ancêtres sont des zombies percés de balles et de marques de tortures. Son folklore porte les masques hideux de l'horreur. Et ses cultures de masses en parodient le versant le plus scandaleux.

Il y a des traductions postmodernes du désir de colmatage de ce vide menaçant, en termes de désire de plus forte démocratie (imparfaite) et de vouloir-vivre ensemble. Avec les musiques rock, on est face à un autre type de traduction de ces mémoires et faits horribles, violents, désordonnés, car les cultures de masses développent aussi une manière de se représenter le fameux vouloir-vivre collectif, cher à Maffesoli, et l'affrontement du futur (Maffesoli, 1981). Les courants rock sont donc aussi des formes diffuses d'appels cyniques ou passionnés, plus sensibles à l'esprit du temps et à la participation. « Putain, putain, c'est vachement bien nous sommes quand même tous des Européens », scandait le groupe belge TCMatic $^{8}$, en 1983 dans cet hymne européen alternatif. Rappelons, pour conclure définitivement cette

\footnotetext{
${ }^{8} \mathrm{Ce}$ groupe belge ( $\mathrm{T}$ et $\mathrm{C}$ renvoyant aux initiales du nom de ses fondateurs) est l'un des groupes phare de la scène postpunk des années 1980. Son principal représentant est devenu ensuite un chanteur rock connu (Arno Hintjens alias «Arno »), une fois que TC Matic s'est dissout en 1986 (après quatre albums et diverses prestations scéniques plus ou moins satisfaisantes). La chanson citée dans cette conclusion (« Putain! Putain ! ») fait partie de l'album Choco, diffusé en 1983 (T.C. Matic ${ }^{\star}$ Choco, Label: EMI - 1 A 064 1191671, Format : Vinyl, LP, Album Country: Benelux).
} 
contribution, que le premier texte fondateur de la tradition européenne demeure celui par qui le souffle divin de la rage et de la fierté combative a circulé dans des mythes, il y a plusieurs millénaires. Sloterdijk nous l'indique discrètement, dès la première page de son livre, en citant la seule phrase rock de l'lliade : « La colère d'Achille, de ce fils de Pélée, chante-la-nous, Déesse... » (Sloterdijk, 2007, p. 9).

\section{Références bibliographiques}

ADORNO Theodor, 1950, «A social critique of radio music », in Bernard Berelson et Janowitz Morris

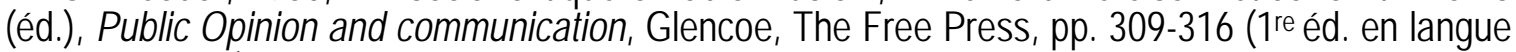
anglaise : 1945).

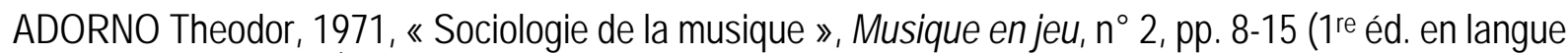
allemande : 1967).

ADORNO Theodor, 2001, Le Caractère fétiche dans la musique et la régression de l'écoute, trad. fr., Paris, Allia (1 $1^{\text {re }}$ éd. en langue allemande : 1973).

ANZIEU Didier et al., 1974, Psychanalyse du génie créateur, Paris, Dunod.

ASSOUN Paul-Laurent, 2006, Le Fétichisme, Paris, PUF.

BARTHES Roland, 1977, Fragments d'un discours amoureux, Paris, Seuil.

BRUCKNER Pascal, 2009, Le Paradoxe amoureux, Paris, Grasset.

CHASTAGNER Claude, 1998, La Loi du rock. Ambivalence et sacrifice dans la musique angloaméricaine, Castelnau-le-Lez, Climats.

DAUFOUY Philippe et SARTON Jean-Pierre, 1972, Pop music rock, Paris, Champ Libre.

DELMAS Yves et GANCEL Charles, 2005, Protest song. La chanson contestataire dans l'Amérique des sixties. Paris, Textuel.

DENIS-CONSTANT Martin, 2006, « Le myosotis, et puis la rose... Pour une sociologie des "musiques de masse" », L'Homme, 1 / 2, n 177-178, pp. 131-154.

DOISE Willem (éd.), 1979, Expériences entre groupes, Paris / La Hayes, Mouton.

DUFOUR Dany-Robert, 2007, Le Divin Marché. La révolution culturelle libérale, Paris, Denoël.

DUFOUR Dany-Robert, 2009, La Cité perverse. Libéralisme et pornographie, Paris, Denoël.

FANON Frantz, 1961, Les Damnés de la terre, Paris, Gallimard.

FERRAND Laure, 2010, La Vie en rock. Une approche compréhensive de la culture rock par ses acteurs : les amateurs, thèse de Doctorat de sociologie (dir. : Prof. Michel Maffesoli), Paris, CEAQ/Université de Paris-V.

FRANÇOIS Stéphane, 2011, «Réflexions sur le mouvement gabberskins. Une première approche sociologique », Les cahiers de psychologie politique; numéro 18 (janvier), URL: http://lodel.irevues.inist.fr/cahierspsychologiepolitique/index.php?id=1747.

FREUD Sigmund, 1970, « Malaise dans la civilisation », trad. fr., in Nouvelle revue de psychanalyse, pp. 9-80 (1re éd. en langue allemande, 1929).

FREUD Sigmund, 1982, Introduction à la psychanalyse, Paris, Payot (1 éd. en langue allemande : 1916).

GIRARD René, 1972, La Violence et le sacré, Paris, Grasset.

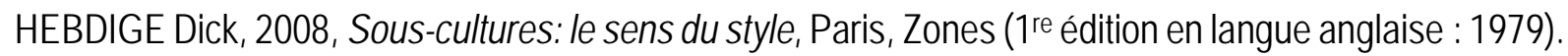

JUNG Carl Gustav, 1964, Dialectique du moi et de l'inconscient, Paris, Gallimard (1're éd. en langue allemande : 1933).

LAPLANCHE Jean et PONTALIS Jean-Bernard, 1967, Vocabulaire de psychanalyse, Paris, PUF. 
MAFFESOLI Michel, 1988, Le Temps des tribus. Le déclin de l'individualisme dans les sociétés de masse, Paris, Klincksieck.

MAFFESOLI Michel, 1981, « La dynamique de l'apparence », L'Homme et la Société, n 59-62 (janvierdécembre), pp. 3-10.

MAFFESOLI Michel, 2007, Le Réenchantement du monde. Une éthique pour notre temps, Paris, La Table Ronde.

MOMBELET Alexis, 2009, Le Metal. Un projet mythologique articulé au jeu et au don, thèse de Doctorat de sociologie, (dir. : Prof. Michel Maffesoli), Paris, CEAQ/Université de Paris-V.

MOSCOVICI Serge, 1981, L'âge des foules. Traité historique de psychologie des masses, Paris, Fayard.

PRICE Jeremy, 2010, « Lonnie Donegan, Rock Island Line, et la corne d'abondance, Volume, vol. VII, $\mathrm{n}^{\circ}$ 2, pp. 115-140.

RIGAUT Philippe, 2009, Continent Dark. Introduction aux subcultures sombres, Paris, Ragage.

ROSOLATO Guy, 1967, «Étude des perversions sexuelles à partir du fétichisme », in AULAGNIERSPAIRANI Piera et al., Le Désir et la perversion, Paris, Seuil, pp. 7-52.

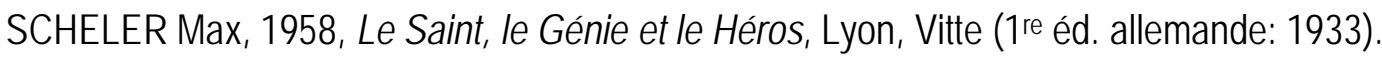

SCHELER Max, 1996, Six essais de philosophie et de religion, Fribourg, Édition universitaires de Fribourg.

SECA Jean-Marie, 1988, Vocations rock. L'état acide et l'esprit des minorités rock, Paris, Klincksieck.

SECA Jean-Marie, 2001, Les Musiciens underground, Paris, PUF.

SECA Jean-Marie (éd), 2007, Musiques populaires underground et représentations du politique, CortilWodon, InterCommunications éditeur.

SECA Jean-Marie, 2009, « Le fil de la devise : les trois dimensions de l'authenticité dans les musiques populaires underground », in Sociétés, numéro thématique Écouter, ressentir et comprendre la musique, numéro 104 / 2, pp. 13-26.

SECA Jean-Marie, 2010, Conduites minoritaires et représentations sociales, Saarbrücken, Éditions universitaires européennes.

SLOTERDIJK Peter, 2007, Colère et temps. Essai politico-psychologique, Paris, Libella Maren Sell (1 éd. en langue allemande : 2006).

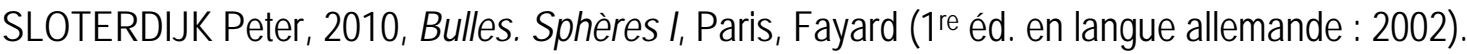

STERNBERG Robert (éd.), 2005, The Psychology of Hate, New York, APA.

STERNBERG Robert et STERNBERG Karin, 2008 (éd.), The Nature of Hate, Cambridge, Cambridge University Press.

STÉVANCE Sophie, 2010, «Reprise et intertextualité musicale. L'hommage à La Bolduc par des compositrices engagées vers l'avant », Volume, vol. VII, $n^{\circ} 2$, pp. 59-81.

THIESSE Anne-Marie, 1999, La création des identités nationales. Europe, XVIle-XXe siècle, Paris, Seuil.

TOOP David, 2008, Ocean of sound. Ambient music, mondes imaginaires et voix de l'éther, Paris, Kargo \& L'Éclat (1re éd. en langue anglaise : 1996).

WALZER Nicolas, 2007, Les Imaginaires satanique et païen : le cas de la musique metal extrême : une volonté de puissance schizomorphe, thèse de Doctorat de sociologie (dir. : Prof. Michel Maffesoli), Paris, CEAQ / Université de Paris-V. 